\title{
Heavy flavors in nucleus-nucleus and proton-nucleus collisions
}

\author{
Marzia Nardi ${ }^{1, a}$ \\ ${ }^{1}$ Istituto Nazionale di Fisica Nucleare - Sezione di Torino \\ Via P. Giuria 1 - 10125 Torino (Italy)
}

\begin{abstract}
A multi-step setup for heavy-flavor studies in high-energy nucleus-nucleus (AA) and proton-nucleus (pA) collisions is presented. The propagation of the heavy quarks in the medium is described in a framework provided by the relativistic Langevin equation, here solved using weak-coupling transport coefficients. Successively, the heavy quarks hadronize in the medium. We compute the nuclear modification factor and the elliptic flow parameter of the final $D$ mesons both in AA and in pA collisions and compare our results to experimental data. ${ }^{\mathrm{b}}$
\end{abstract}

\section{Introduction}

The primary goal of the ongoing heavy-ion collision experiments at the Relativistic Heavy Ion Collider (RHIC) and at the Large Hadron Collider (LHC) is to create and study a new state of matter, the Quark-Gluon Plasma (QGP), where quarks and gluons are no longer confined, but can freely move over distances much larger than the typical hadronic size.

A nucleus-nucleus collision is a very complicated process undergoing several stages: right after the first interaction between the incident nuclei (or proton and nucleus) a dense system of partons is produced, which shortly reach a thermal equilibrium (at least locally) and form the QGP. It then expands, cools down and decades into a system of interacting hadrons. The hadronic interactions cease when the system, still expanding and colling, is too dilute. The final hadrons (or their decay products) are then detected in the experimental apparatus.

Many observables has been proposed to study the properties of the QGP. Among them, the heavy quarks (charm and beauty) play a special role, for the following reasons:

- their mass $M$ is much larger than $\Lambda_{Q C D}$ : it is possible to compute cross sections and $p_{T}$ spectra with perturbative techiques from the QCD theory (next-to-lead order calculations are, presently, the "status of the art");

- $M$ in also larger than the average temperature of the medium formed in the collision: their thermal production is expected to be negligible both in the plasma and, even more, in the hadronic phase;

- they are created at the very beginning of the collision between the two incident nuclei (or proton and nucleus) and witness all the subsequent evolution;

\footnotetext{
a e-mail: nardi@to.infn.it

${ }^{b}$ Work done in collaboration with A. Beraudo, A. De Pace, M. Monteno and F. Prino (INFN, Torino)
} 
- $M \gg g T$, with $g T$ being the typical momentum exchange in the collision with the plasma particle: many soft scattering are needed to change significantly the momentum (and trajectory) of the heavy quark $^{1}$; therefore heavy quarks do not thermalize with the medium in the later stage, they do not loose "memory" of their initial momentum and energy and carry precious information about the QGP phase.

In a series of papers [1-4] over the last few years we developed a complete setup (referred to as POWLANG) for the study of heavy flavour observables in high-energy nucleus-nucleus (AA) collisions, describing the initial hard production of the $Q \bar{Q}$ pairs and the corresponding parton-shower stage through the POWHEG-BOX package [5, 6] and addressing the successive evolution in the plasma through the relativistic Langevin equation. Here, following Ref. [4], we supplement our numerical tool by modeling the hadronization of the heavy quarks accounting for the presence of a surrounding medium made of light thermal partons feeling the collective flow of the local fluid cell. Moreover, we present our first (preliminary) results for proton-nucleus (pA) collisions at LHC energies.

\section{Heavy flavour in proton-proton collisions}

Because of their large mass, the initial production of $c$ and $b$ quarks is a short-distance process involving a large-momentum transfer, described by perturbative QCD (pQCD). For this purpose we rely on a standard $\mathrm{PQCD}$ public tool, namely POWHEG-BOX, in which the hard $Q \bar{Q}$ event is interfaced with a shower stage described by PYTHIA [7], to include the effects of Initial- and Final-State Radiation $[1,2]$.

Experimental data obtained in proton-proton ( $\mathrm{pp}$ ) collisions can be exploited to validate the theoretical calculations used to simulate the initial hard $Q \bar{Q}$ production. In our setup, the heavy quarks are created in pairs by the POWHEG-BOX event generator. Their momenta are not back-to-back neither along the beam-axis, due to the different Bjorken- $x$ carried by the partons taking part in the hard event, nor in the azimuthal plane, due to the gluon radiation occurring during the hard process or the shower stage and also to the intrinsic $k_{T}$-broadening one can include in the simulation. Eventually, heavy-quark hadronization and the final decays of the $D(B)$ mesons are simulated with PYTHIA, which is also used to describe the parton shower stage.

In Fig. 1 we show some results for $p p$ collisions. In the left panel, the inclusive $p_{T}$ distribution of $D^{0}$ mesons [3] is compared to experimental data measured by the ALICE collaboration [8]. Also shown is the FONLL systematic uncertainty band [9]. The right panel shows $D-h$ azimuthal correlations compared to preliminary ALICE data $[10,11]$, for two different $p_{T}$-intervals of the charmed meson. In our simulation $D^{0}, \bar{D}^{0}$ and $D^{ \pm}$are used as trigger particles and the light hadrons are limited to charged pions and kaons, protons and antiprotons, excluding the weak decays of $\Lambda$ and $K^{0}$. Any $D$-meson is correlated with all the light hadrons (except its own decay products) created in the same event. The near-side peak takes contribution both from correlations present at the partonic level (from $Q \bar{Q}$ pairs arising from gluon splitting) and from hadrons coming from the fragmentation of the same string of the parent heavy quark. Our results include also the simulation of the Underlying Event due to Multiple Parton Interactions, performed with PYTHIA 6.4, which gives rise to the pedestal observed in Fig. 1.

The agreement between the results of POWHEG+PYTHIA event generator and the experimental data is quite good: this makes us confident that the production process is reasonably well descripted and can be used as a starting point for the simulation of AA and pA collisions.

\footnotetext{
${ }^{1}$ For realistic temperature $g \sim 2$, so the present condition, at least in the initial stage of the evolution, is only marginally fulfilled by charm quarks.
} 

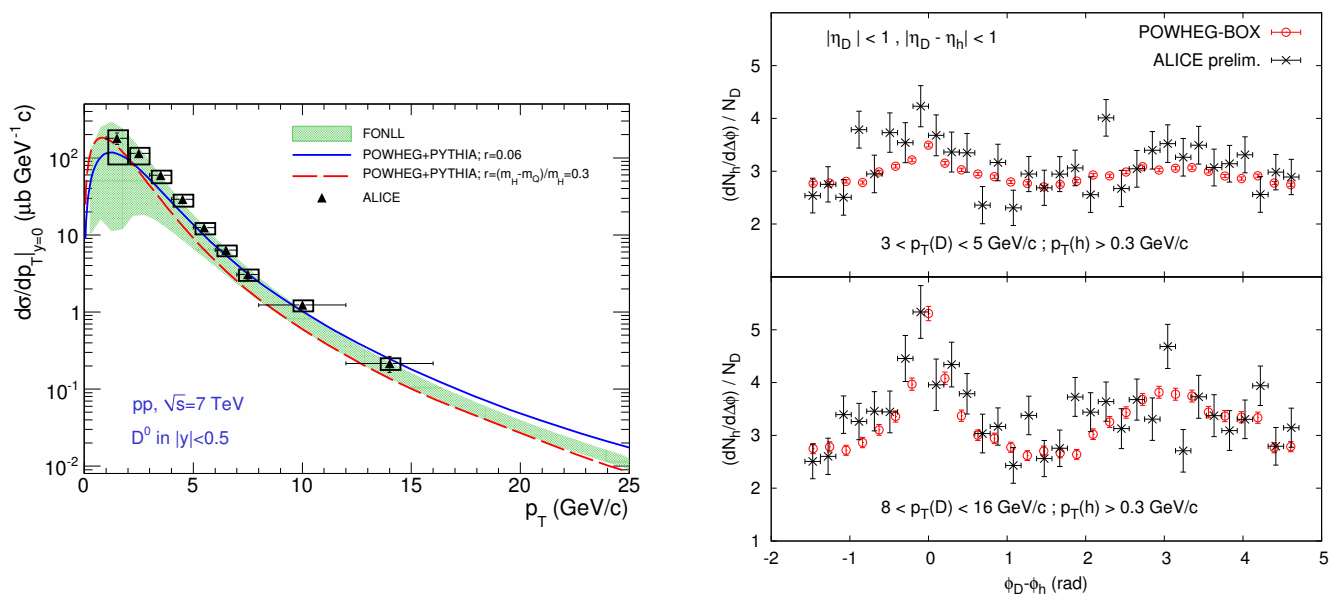

Figure 1. Results for $p p$ collisions at $\sqrt{s}_{N N}=7 \mathrm{TeV}$ compared to experimental data by the ALICE Collab. $[8,10,11]$. Left: $p_{T}$ spectrum for $D^{0}$ mesons. Right: azimuthal $D-h$ correlations for different $p_{T}$-cuts.

\section{Heavy flavour in pA and AA collisions: initial conditions}

In nuclear collisions the $c \bar{c}$ or $b \bar{b}$ production given by the pQCD calculation must be modified in two ways: $i$ ) the nuclear parton distribution functions should be corrected for shadowing or antishadowing effects (we have adopted here the EPS09 scheme [12]); $i$ ) the colliding partons acquire, on average, a larger transverse momentum during the crossing of the two nuclei (Cronin effect), which can be described by a Glauber calculation [2].

Moreover, their initial positions are distributed in the transverse plane according to the density of nucleon-nucleon collision, as computed in the (optical) Glauber model.

The heavy quarks created in nuclear collisions propagate in a strongly interacting and non-static medium, whose properties and evolution is described through hydrodynamical calculations, performed with the viscous 2+1 code of Ref. [13] (for the AA case), or with ECHO-QGP [14] (for the $\mathrm{pA}$ case, in $2+1$ dimensions for simplicity). The assumption of longitudinal boost-invariance (implicit in the $2+1$ description) restricts the validity of our calculation to a limited region around mid-rapidity.

While for the initial conditions in AA collisions one can adopt the smooth results provided by an optical-Glauber calculation, in a pA collision the event-by-event fluctuations in the initial state are extremely important, since they are the main source of anisotropic flow in the final state.

We assumed that each nucleon-nucleon collisions, occurring at the transverse coordinate $\mathbf{x}_{i}$ in a give initial distribution (the single nucleons being randomly located with a distribution probability given by a realistic nuclear density), deposit some entropy in the transverse plane, distributed according to a gaussian form centered around the scattering position and depending on the smearing parameter $\sigma$. For the numerical calculation we tested two values of $\sigma: 0.2$ and $0.4 \mathrm{fm}$.

The total entropy distribution thus results in:

$$
s(\mathbf{x})=\frac{K}{2 \pi \sigma^{2}} \sum_{i=1}^{N_{\text {coll }}} \exp \left[-\frac{\left(\mathbf{x}-\mathbf{x}_{i}\right)^{2}}{2 \sigma^{2}}\right]
$$



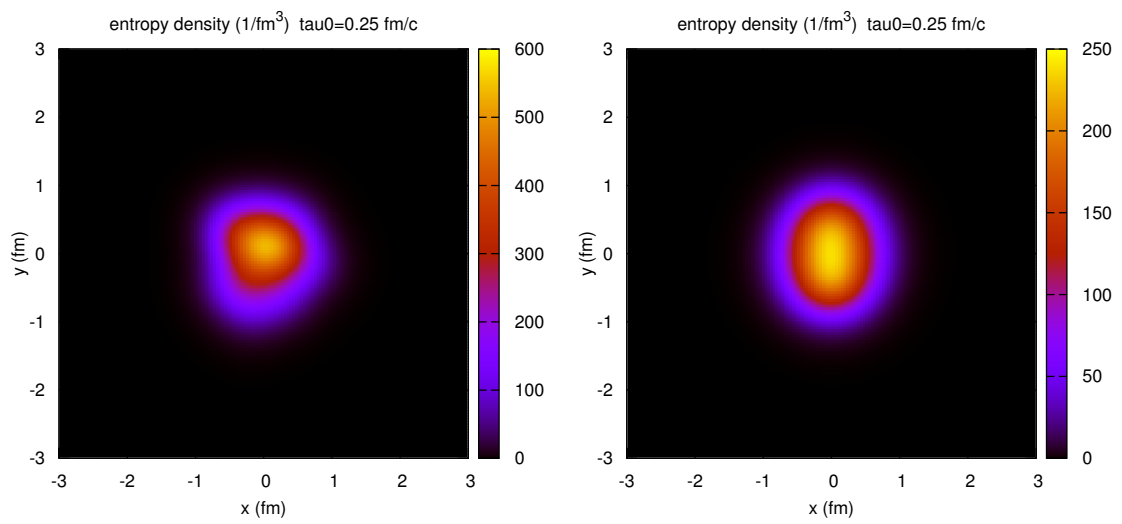

Figure 2. The initial entropy-density profile for a single $\mathrm{p}-\mathrm{Pb}$ collision at $\sqrt{s}_{N N}=5.02 \mathrm{TeV}$ (left panel) and the result of the weighted average of the $0-20 \%$ most central events (right panel).

The multiplicative constant $K$ can be fixed, for instance, by matching the initial entropy density calculated with the Glauber Model in the optical approximation and in the Monte Carlo simulation. The initial eccentricity, which translate into a non-vanishing elliptic flow [15], can be then evaluated as (the brackets denote the average in the transverse plane, with the entropy density in Eq. 1 as a weight):

$$
\epsilon_{2}=\frac{\sqrt{\left\{y^{2}-x^{2}\right\}^{2}+4\{x y\}^{2}}}{\left\{y^{2}+x^{2}\right\}} .
$$

Since a full event-by-event simulation with our hydro+transport setup would be really demanding, for the huge computing and storage resources required, we evaluate a realistic average background as follows: for a given centrality class we average over all the events of the considered percentile (based on the number of $N_{\text {part }}$ ); After rotating each of them by the event-plane angle $\psi_{2}$, we compute an average entropy-density distribution from which a realistic average eccentricity $\epsilon_{2}$ can be obtained. In Fig. 2 we shown an example of a single event (left) and the average distribution (right), for a p-Pb collision at $\sqrt{s}_{N N}=5.02 \mathrm{TeV}$.

\section{Propagation of the Heavy Quarks through the medium}

The time evolution of the heavy-quark phase-space distribution $f_{Q}(t, x, \boldsymbol{p})$ can be described by the Boltzmann equation:

$$
\frac{\mathrm{d}}{\mathrm{d} t} f_{Q}(t, x, \boldsymbol{p})=C\left[f_{Q}\right]
$$

$C\left[f_{Q}\right]$ is the Boltzmann collision integral, taking into account losses and gains due to single-particle collisions [16]:

$$
C\left[f_{Q}\right]=\int \mathrm{d} \boldsymbol{k}\left[w(\boldsymbol{p}+\boldsymbol{k}, \boldsymbol{k}) f_{Q}(\boldsymbol{p}+\boldsymbol{k})-w(\boldsymbol{p}, \boldsymbol{k}) f_{Q}(\boldsymbol{p})\right],
$$

where $w(p, k)$ is the transition rate for a heavy quark changing its momentum from $\boldsymbol{p}$ to $\boldsymbol{p}-\boldsymbol{k}$. 
Neglecting $x$ - and mean field dependence of $f_{Q}$ and expanding the collision integral for small momentum exchange (Landau approximation [17]) the collision integral can be expressed as:

$$
C\left[f_{Q}\right] \simeq \int \mathrm{d} \boldsymbol{k}\left[k^{i} \frac{\partial}{\partial p^{i}}+\frac{1}{2} k^{i} k^{j} \frac{\partial^{2}}{\partial p^{i} \partial p^{j}}\right]\left[w(\boldsymbol{p}, \boldsymbol{k}) f_{Q}(t, \boldsymbol{p})\right] ;
$$

the Boltzmann equation reduces to the Fokker-Planck equation:

$$
\frac{\partial}{\partial t} f_{Q}(t, \boldsymbol{p})=\frac{\partial}{\partial p^{i}}\left\{A^{i}(\boldsymbol{p}) f_{Q}(t, \boldsymbol{p})+\frac{\partial}{\partial p^{j}}\left[B^{i j}(\boldsymbol{p}) f_{Q}(t, \boldsymbol{p})\right]\right\}
$$

where

$$
\begin{aligned}
A^{i}(\boldsymbol{p}) & =\int \mathrm{d} \boldsymbol{k} k^{i} w(\boldsymbol{p}, \boldsymbol{k})=A(p) p^{i} \\
B^{i j}(\boldsymbol{p}) & =\frac{1}{2} \int \mathrm{d} \boldsymbol{k} k^{i} k^{j} w(\boldsymbol{p}, \boldsymbol{k})=B_{0}(p)+\left(\delta^{i j}-\hat{p}^{i} \hat{p}^{j}\right) B_{1}(p) .
\end{aligned}
$$

The problem is reduced to the evaluation of three transport coefficients: a friction term $A(p)$ and two terms describing the momentum broadening $B_{0}(p)$ and $B_{1}(p)$.

The Fokker-Planck equation can be then recast into a form suitable to follow the dynamics of each individual quark: the Langevin equation

$$
\frac{\Delta p^{i}}{\Delta t}=-\eta_{D}(p) p^{i}+\xi^{i}(t)
$$

with the properties of the noise encoded in

$$
\left\langle\xi^{i}\left(\boldsymbol{p}_{t}\right) \xi^{j}\left(\boldsymbol{p}_{t^{\prime}}\right)\right\rangle=b^{i j}\left(\boldsymbol{p}_{t}\right) \frac{\delta_{t t^{\prime}}}{\Delta t} \quad b^{i j}(\boldsymbol{p})=\kappa_{\|}(p) \hat{p}^{i} \hat{p}^{j}+\kappa_{\perp}(p)\left(\delta^{i j}-\hat{p}^{i} \hat{p}^{j}\right) .
$$

The transport coefficients $\kappa_{\perp}$ and $\kappa_{\|}$can be calculated within a weakly-coupled scenario: perturbative QCD and "Hard Thermal Loop" (HTL) approximation, as done in Ref. [1]. We note that the transport coefficients computed with pQCD+HTL have a strong dependence on the momentum $p$ of the heavy quark and on the temperature $T$ of the system.

The Langevin approach is a very convenient numerical tool and allows one to establish a link between observables and transport coefficients derived from QCD. However, it was derived starting from a soft-scattering expansion of the collision integral $C[f]$ truncated at second order (friction and diffusion terms): in Ref. [16] it was found that this approximation is excellent for $b$-quarks for all the evolution of the system, while for $c$ - quarks it is valid, in a short time interval of a few $\mathrm{fm} / \mathrm{c}$ after the initial collision, only for small momenta.

Having all the ingredients, we can now procede to simulate the heavy-quark propagation in the medium. This occurs through several steps, repeated iteratively until hadronization:

1. a heavy quark with momentum $p$ is located in a position $x$ at a given time $t$ : the hydrodynamical simulation tells us the conditions (temperature $T(x, t)$, energy density $\varepsilon(x, t)$, four-velocity $\left.u^{\mu}(\boldsymbol{x}, t), \ldots\right)$ of the surrounding fluid cell;

2. we perform a Lorentz boost in the local fluid rest-frame and set the transport coefficients using the temperature and the momentum $\tilde{p}$ of the quark (in this reference system);

3. we use the Langevin equation to compute the new quark momentum $\tilde{p}^{\prime}$ and its new position $\tilde{\boldsymbol{x}}^{\prime}$ at the time $t+\Delta t$ 
4. we perform another Lorentz boost back to the laboratory frame; we check whether the surrounding fluid cell is in the QGP phase (i.e. its temperature is higher than the deconfining temperature $T_{d e c}$ : if so, we restart from step 1 , otherwise the quark hadronizes.

The time step used in our simulation is $\Delta t=0.02 \mathrm{fm} / \mathrm{c}$, for the deconfining temperature $T_{\text {dec }}$ we have tested two values: $T_{d e c}=155 \mathrm{MeV}$ (the value quoted in Lattice calculation for the QGP-hadron transition) and $T_{d e c}=170 \mathrm{MeV}$, allowing the heavy quarks to form bound states with light quarks which may survive, in a small temperature range, in the deconfined phase [18].

\section{Hadronizations of the Heavy Quarks}

Finally, after the propagation through the dense medium, the heavy quarks decouple and hadronize. In the pp case, the hadronization is performed with PYTHIA, while for the nuclear collisions we introduce a new simple model to take into account the effect of the thermalized medium.

Once a heavy quark $Q$, during its stochastic propagation in the fireball, has reached a fluid cell below the decoupling temperature $T_{\mathrm{dec}}$, it is forced to hadronize. One extracts then a light antiquark $\bar{q}$ (up, down or strange, with relative thermal abundances dictated by the ratio $m / T_{\mathrm{dec}}$ ) from a thermal momentum distribution corresponding to the temperature $T_{\mathrm{dec}}$ in the local rest frame of the fluid; the local fluid four-velocity $u^{\mu}$ allows one to boost the momentum of $\bar{q}$ to the laboratory frame. A string is then constructed joining the endpoints given by $Q$ and $\bar{q}$ and is then given to PYTHIA 6.4 to simulate its fragmentation into hadrons (and their final decays). In case the invariant mass of the string is not large enough to allow its decay into at least a pair of hadrons the event is resampled, extracting a new thermal parton to associate to the heavy quark.

With this hadronization scheme we can first of all provide a realistic estimate of the role of the thermal light quarks to explain peculiar features of the $D$ meson spectra at low and moderate $p_{T}$; secondly, the complete information on all the final state particles arising from the fragmentation of the strings allows us to provide theory predictions for observables like $D-h, e-h, e^{+}-e^{-} \ldots$ angular correlations to be compared to existing data and possibly used as a guidance to future measurements.

Notice that further possible interactions in the hadronic phase, which might enhance the elliptic flow, are here neglected: we plan to include them in our future work.

\section{Results: AA collisions}

In Fig. 3 we show some POWLANG outcomes for the $R_{A A}$ of $D^{0}$ mesons in central $(0-10 \%) \mathrm{Au}-\mathrm{Au}$ collisions at $\sqrt{s_{N N}}=200 \mathrm{GeV}$. HTL transport coefficients are employed and the difference between the two hadronization schemes (here taken to occur at $T_{\mathrm{dec}}=170 \mathrm{MeV}$, either with vacuum fragmentation or with in-medium string fragmentation, are clearly visible: in the second case the radial flow of the light thermal parton leads to the development of a bump around $p_{T} \sim 1.5 \mathrm{GeV}$ in qualitative agreement with the experimental data. Also shown for comparison is the result for the limiting scenario in which charmed particles reach full kinetic equilibrium with the medium [4]. Our results are compared to STAR data[19].

The ALICE Collaboration has recently released the data for the in-plane/out-of-plane nuclear modification factors of $\mathrm{D}$ mesons in semi-central (30- 50\%) Pb-Pb collisions [20]. Experimental findings are displayed in Fig. 4 and compared to the transport predictions of the POWLANG setup: also in this case model results with in-medium fragmentation look in better agreement with the experimental data. The theoretical results are given for two different values of the deconfining temperature: we notice that the nuclear modification factor $R_{A A}$ is not very sensitive to the value of $T_{\mathrm{dec}}$. 


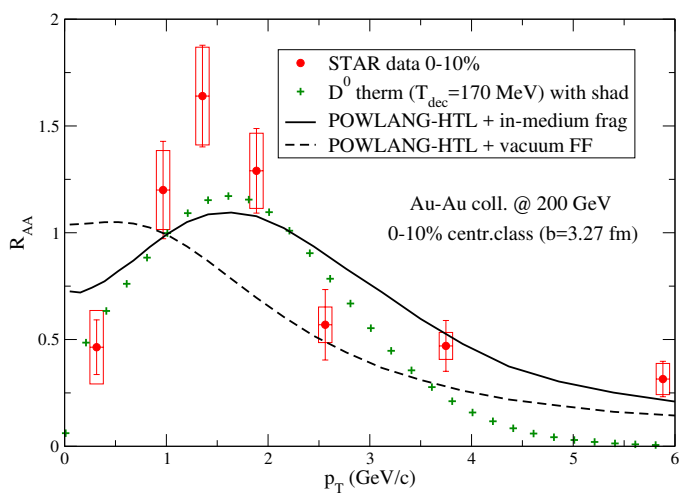

Figure 3. The $R_{A A}$ of $D^{0}$ mesons in central $\mathrm{Au}-\mathrm{Au}$ collisions at $\sqrt{s_{N N}}=200 \mathrm{GeV}$. POWLANG results obtained with HTL transport coefficients and a decoupling temperature $T_{\mathrm{dec}}=170 \mathrm{MeV}$ are plotted. Also shown for comparison is the extreme case of full kinetic thermalization of $D$ mesons. Theory curves are compared to STAR data [19]
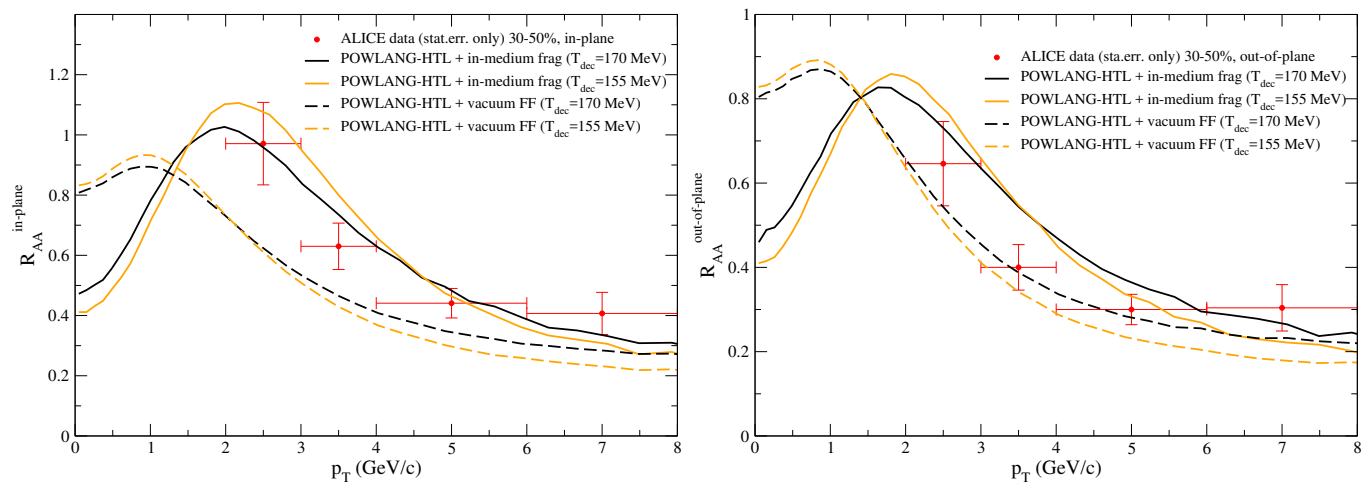

Figure 4. $R_{A A}$ in-plane (left) and out-of-plane (right) of $D$ mesons. ALICE data in the $30-50 \%$ centrality class [20] are compared to POWLANG results obtained with different hadronization mechanisms (in-medium and vacuum fragmentation) and decoupling temperatures $\left(T_{\mathrm{dec}}=170 \mathrm{MeV}\right.$ and $\left.155 \mathrm{MeV}\right)$.

In Fig. 5 we address the $v_{2}$ of $D$ mesons in semicentral (30-50\%) Pb-Pb collisions at $\sqrt{s_{N N}}=2.76$ $\mathrm{TeV}$ at the LHC. The effect of the new procedure for in-medium hadronization through string fragmentation is clearly visible: while POWLANG outcome with standard in-vacuum fragmentation of charm largely underpredicts the observed $v_{2}$, the additional flow acquired from the light thermal partons move the theory curves with in-medium hadronization closer to the experimental data measured by the ALICE Collaboration [21]. The value chosen for the decoupling temperature is $T_{\mathrm{dec}}=155$ $\mathrm{MeV}$; we observed that $v_{2}$ is more sensitive to $T_{\mathrm{dec}}$ than other observables, the lower value seeming to be preferred by the data: this agrees with the expectation that the elliptic flow needs more time to fully develop with respect to the quenching of the spectra. This plot also shows how a full kinetic thermalization up to large values of $p_{T}$ is disfavoured by the data.

Finally we decide to address more differential observables like angular correlations between heavy flavour particles (or their decay products) and the charged hadrons produced in the same collision. In Fig. 6 we display our results for $D-h$ azimuthal correlations. All figures are obtained with weakcoupling HTL transport coefficients in the QGP phase. In general one observes a strong suppression of the away-side peak around $\Delta \phi=\pi$. Depending on the cuts imposed on the trigger particles ( $D$-mesons) and on the associated hadrons this can be mostly due either to the energy loss (moving particles below 


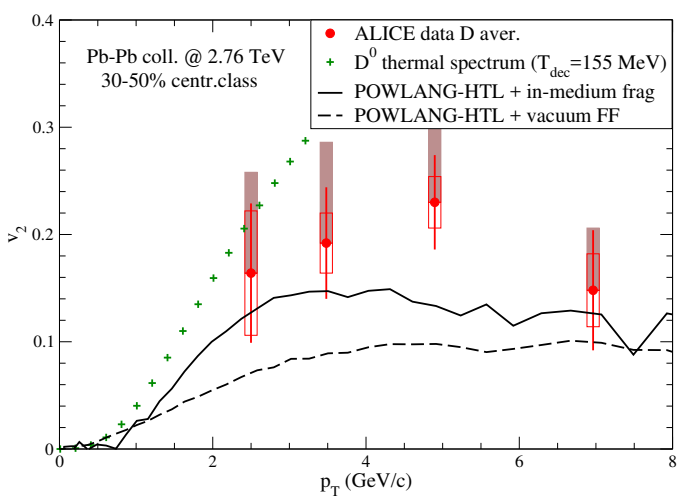

Figure 5. The $v_{2}$ of $D$ mesons in $\mathrm{Pb}-\mathrm{Pb}$ collisions at $\sqrt{s_{N N}}=2.76 \mathrm{TeV}$. POWLANG results (with HTL transport coefficients) with in-vacuum and in-medium HQ fragmentation at the decoupling temperature $T_{\mathrm{dec}}=155 \mathrm{MeV}$ compared to ALICE data [21] in the $30-50 \%$ centrality class and to the limit of kinetic thermalization.
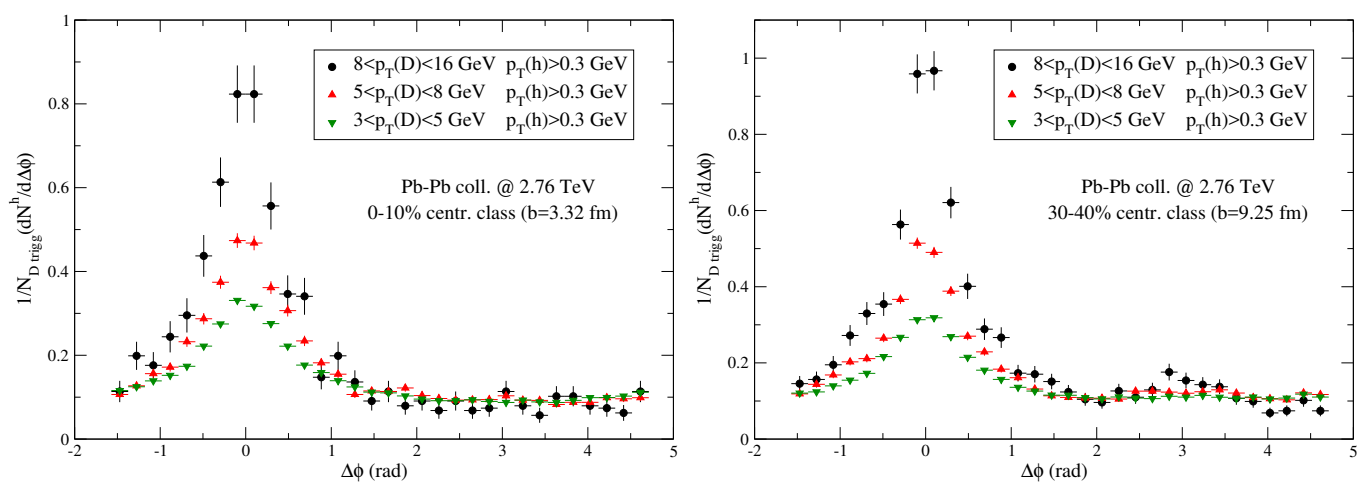

Figure 6. $D-h$ correlations in $\mathrm{Pb}-\mathrm{Pb}$ collisions at $\sqrt{s_{\mathrm{NN}}}=2.76 \mathrm{TeV}$ for various $p_{T}$ cuts on the trigger particle and different centrality classes: $0-10 \%$ (left) and $30-40 \%$ (right).

the $p_{T}$-cut) or to the angular decorrelation (moving particles away from $\Delta \phi=\pi$ ) of the parent heavy quark.

\section{Results: pA collisions}

For quite some time, it was commonly believed that in pA collision the formation of QGP can not occur, because the small size of the system does not allow to reach a sufficiently dense system in thermal equilibrium. Such measurements were considered just a benchmark to estimate cold nuclearmatter effects. However, recent experimental data on central p-Pb collisions at the LHC and d-Au (and ${ }^{3} \mathrm{He}-\mathrm{Au}$ ) at RHIC may suggest the formation of a medium with collective behaviour even in such small systems.

It is therefore very important to make accurate simulations of these processes in order to give a correct interpretation of current experimental data.

We show some preliminary POWLANG results for $c$-quarks in $\mathrm{p}$-Pb collisions at LHC. Work is still in progress to extend to calculation to the bottom quarks and to simulate d-Au collisions at RHIC.

In Fig. 7 we show how the formation of a hot deconfined medium in the p-Pb collision at $\sqrt{s_{N N}}=$ $5.02 \mathrm{TeV}$ affects the heavy quark and hadron spectra, by modifying their propagation and subsequent 

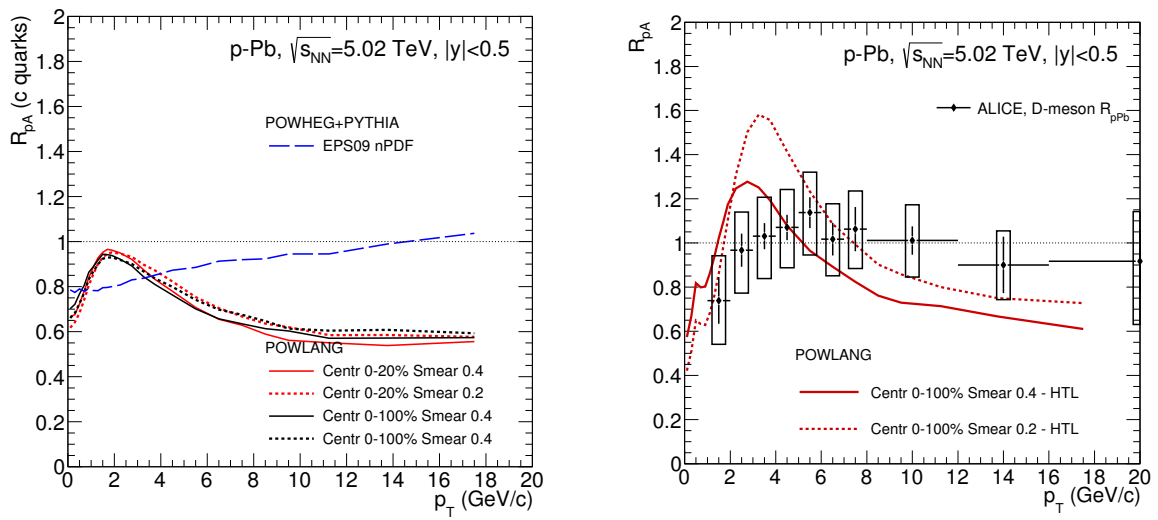

Figure 7. The nuclear modification factor of $c$-quarks (left) and for $D$ mesons (right) in p-Pb collisions at $\sqrt{s_{N N}}=$ $5.02 \mathrm{TeV}$. POWLANG results (with HTL transport coefficients) with in-medium heavy-quark fragmentation, for $\sigma=0.2$ and $0.4 \mathrm{fm}$. Experimental data from Ref. [22]

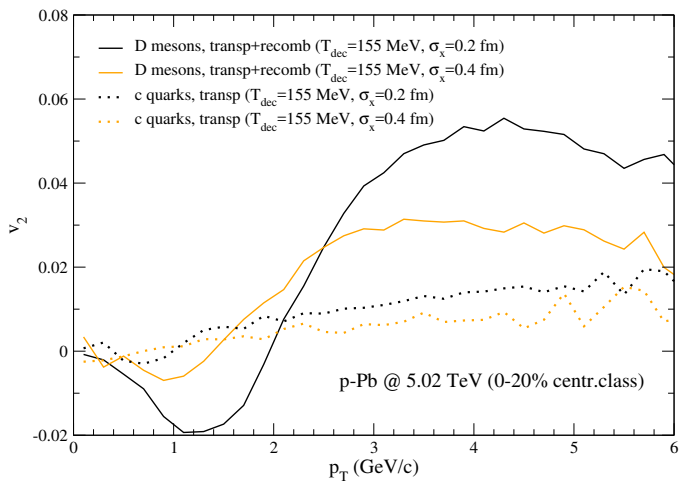

Figure 8. The $v_{2}$ of $\mathrm{D}$ mesons in $\mathrm{p}-\mathrm{Pb}$ collisions at $\sqrt{s_{N N}}=5.02$ TeV. POWLANG results (with HTL transport coefficients) with in-medium heavy-quark fragmentation, for $\sigma=0.2$ and $0.4 \mathrm{fm}$. The $v_{2}$ coefficients for c-quarks alone are plotted for comparison.

hadronization. The left panel shows the nuclear modification factor for $c$-quarks: the blue, dashed line shows the result for the quarks produced by initial-state interaction, according to the EPS09 parton distribution functions (before the propagation in the expanding medium); the dotted and solid lines give the analogous result for the charm quarks after the propagation (both for central and minimum bias collisions, with two different choices of the smearing parameter od Eq. 1). The difference between the EPS09 curve and the other ones is a clear evidence of the effect of Langevin dynamics on the $c$ quark even in such a small system. In the right panel we show the result for the final $D$ meson, with the hadronization occurring in the medium as explained in Sec. 5. The peak at $p_{T}=3 \div 4 \mathrm{GeV} / \mathrm{c}$ (more pronounced for $\sigma=0.2 \mathrm{fm}$ ) is a consequence of the radial flow of light quarks inherited by the meson. The POWLANG result is compatible, within errorbars, with the experimental data measured by the ALICE Collaboration [22].

In Fig. 8 the elliptic flow coefficient $v_{2}$ is plotted, both for $c$-quarks (dotted lines) and for $D$ mesons, for a deconfining temperature of $155 \mathrm{MeV}$. Again, the contribution of the light-quark flow, inherited by the charmed meson in the hadronization process, is crucial to enhance the $v_{2}$. 


\section{CONCLUSIONS}

The simple model to describe heavy quark hadronization in the presence of a hot deconfined medium (a Quark-Gluon Plasma) has considerably improved the agreement of the POWLANG results with the experimental data at RHIC and LHC energies. In particular, results for the nuclear modification factors and the elliptic flow of D mesons have been presented.

Recently, signatures of final-state medium effects were observed also in pA collisions: we have presented some preliminary results for $\mathrm{p}-\mathrm{Pb}$ collisions at LHC energies, and we plan to continue this study in more detail in the next future.

\section{References}

[1] A. Beraudo, A. De Pace, W.M. Alberico, A. Molinari, Nucl. Phys. A 831, 59 (2009)

[2] W.M. Alberico, A. Beraudo, A. De Pace, A. Molinari, M. Monteno, M. Nardi, F. Prino, Eur. Phys. J. C 71, 1666 (2011)

[3] W.M. Alberico, A. Beraudo, A. De Pace, A. Molinari, M. Monteno, M. Nardi, F. Prino, M. Sitta, Eur.Phys.J. C73, 2481 (2013)

[4] A. Beraudo, A. De Pace, M. Monteno, M. Nardi and F. Prino, Eur.Phys.J. C75, 121 (2015) 3

[5] S. Frixione, P. Nason, G. Ridolfi, JHEP 0709 (2007) 126.

[6] S. Alioli, P. Nason, C. Oleari and E. Re, JHEP 1006 (2010) 043.

[7] T. Sjostrand, S. Mrenna and P.Z. Skands, JHEP 0605 (2006) 026.

[8] ALICE Collaboration (B.I. Abelev et al.), J. High Energy Phys. 1201, 128 (2012)

[9] M. Cacciari, M. Greco, P. Nason, J. High Energy Phys. 9805, 007 (1998); J. High Energy Phys. 0103, 006 (2001)

[10] S. Bjelogrlić (ALICE Collab.), Nucl. Phys A 931, 563 (2014)

[11] A. Rossi (ALICE Collab.), Nucl. Phys A 932, 51 (2014)

[12] K.J. Eskola, H. Paukkunen, C.A. Salgado, J. High Energy Phys. 0904, 065 (2009)

[13] P. Romatschke, U. Romatschke, Phys. Rev. Lett. 99, 172301 (2007).

[14] ECHO-QGP project: http://theory.fi.infn.it/echoqgp

[15] H. Niemi, K. J. Eskola and R. Paatelainen, arXiv:1505.02677 [hep-ph].

[16] V. Greco et al., Phys. Rev. C 90 (2014) 4, 044901

[17] B. Svetitsky Phys. Rev. D 37, 2484 (1988)

[18] W. M. Alberico et al. Phys. Rev. D 72 (2005) 114011.

[19] L.Adamczyk et al. (STAR Collab.), arXiv:1404.6185 [nucl-ex].

[20] ALICE Collaboration (B. Abelev et al.) 2014 Phys. Rev. C 90034904

[21] B. Abelev (ALICE Collaboration), Phys. Rev. Lett. 111 (2013) 102301.

[22] B. B. Abelev (ALICE Collaboration), Phys. Rev. Lett. 113 (2014) 23, 232301. 